\title{
Le dessin comme méthode : du portrait à la scène
}

\section{Drawing as method: from portrait to scene}

\author{
Cécile Dupin De Saint Cyr-Heckel ${ }^{1}$, Viviane Couzinet $^{2}$, Isabelle Fabre ${ }^{3}$ \\ ${ }^{1}$ Laboratoire d'Etudes et recherches appliquées en sciences sociales, Université Toulouse III-Paul Sabatier, \\ Cecile.Heckel@ac-montpellier.fr \\ ${ }^{2}$ Laboratoire d'Etudes et recherches appliquées en sciences sociales, Université Toulouse III-Paul Sabatier, \\ viviane.couzinet@iut-tlse3.fr \\ ${ }^{3}$ Education, Formation, Travail, Savoirs, Université de Toulouse II-Jean Jaurès, isabelle.fabre@ensfea.fr
}

\begin{abstract}
RÉSUMÉ. Le portrait textuel ou iconographique est utilisé dans de nombreuses disciplines afin de mettre en lumière les rôles tenus par des personnes ou des groupes. Cependant il reste peu convoqué comme méthode d'investigation en sciences de l'information et de la communication. A partir d'observations conduites auprès d'élèves de collège auxquels il a été demandé de se dessiner, il est possible de considérer que l'autoportrait, des dessins de l'observateur et de pairs peuvent contribuer à révéler des pratiques. Une grille interprétative appuyée sur le vocabulaire de langages documentaires facilite l'interprétation. Ainsi du portrait à la scène les dessins complètent d'autres méthodes d'enquête.

ABSTRACT. The textual or iconographic portrait is used in many disciplines to highlight the roles held by individuals or groups. However, it remains little used as a method of investigation in information and communication sciences. Based on observations made with secondary school pupils who have been asked to draw up, it is possible to consider that the self-portrait, drawings by the observer and by peers can help to reveal practices. An interpretative grid based on the vocabulary of documentary languages facilitates interpretation. Thus from the portrait to the scene the drawings complete other methods of investigation.

MOTS-CLÉS. Méthode du dessin, portrait, scène, sciences de l'information et de la communication.

KEYWORDS. Drawing method, portrait, scene, Information and communication sciences.
\end{abstract}

\section{Introduction}

Le portrait est largement utilisé en recherche afin d'analyser les rôles tenus par des personnages ou des groupes marquants en histoire (une figure), en littérature (un auteur) ou en art (un artiste). On le retrouve également dans l'étude de personnalités qui par leur action ou la richesse de leur apport théorique ont imprégné une discipline. En sciences de l'information et de la communication (SIC) le chapitre français de l'International Society of Kowledge Organization, en 2017, puis la revue Les Cahiers du numérique ${ }^{1}$, en 2019, leur ont rendu hommage. Ces portraits sont souvent des représentations textuelles, les représentations iconographiques étant plutôt des œuvres d'artistes. Cependant, dans les unes comme dans les autres, l'objectif est de rapporter et d'interroger par l'écrit, par le trait ou par la peinture, ce qui définit un caractère, des actes et une manière d'agir, une pensée et la construction de connaissances, des engagements dans des situations données. Le portrait est ainsi un genre artistique et un genre d'écrit de recherche qui, tout particulièrement pour ce dernier, se penche sur le passé et participe à l'élaboration de l'histoire des disciplines. Il permet aussi de mettre en lumière des acteurs au travers d'un travail empirique sur leurs œuvres et de conduire une réflexion sur la place qu'ils occupent dans un groupe social donné. La part de la biographie y est importante.

\footnotetext{
${ }^{1}$ MUSTAFA EL HADI W. (dir.), «Fondements épistémologiques et théoriques de la science de l'information-documentation », Actes du $11^{e}$ colloque ISKO France 2017, ISTE Editions, Londres, 2017 ; Appel à articles : CoUZINET V., MuSTAFA EL HADI W., PAPY F. (dir.), «De quelques théoriciens et quelques praticiens de l'information-documentation », Les Cahiers du numérique, 2019.
} 
Par ailleurs le dessin est utilisé par certains chercheurs comme matériaux d'analyse des représentations de populations spécifiques. Isabelle Fabre, par exemple, a étudié la perception de l'aménagement d'espaces documentaires à partir de plans réalisés par les usagers (Fabre, 2006, 2008). Cependant ces représentations, si elles sont une projection d'une manière de percevoir un lieu, sont essentiellement des représentations d'un cadre d'action. Qu'en est-il de la représentation du sujet par lui-même? Qu'en est-il de sa représentation comme être en action ? Est-ce que la perception de «soi agissant » diffère de celle de soi perçu par les autres ? Et comment analyser ces représentations de soi en SIC sans tomber dans l'analyse psychologique ? Peut-on mettre à contribution des outils d'analyse utilisés par les professionnels de l'information-documentation pour éviter cette dérive ? Faisant appel au dessin et à un outil de traitement de l'information, les langages documentaires, afin de repérer les éléments signifiants ce questionnement ambitionne de proposer une méthode d'investigation qui s'appuie sur les acquis d'autres disciplines et qui les adapte à l'examen d'objets étudiés par les SIC.

Après avoir examiné les différents travaux qui utilisent le portrait comme objet ou comme mode d'investigation scientifique nous exposerons notre manière d'aborder les questions posées. A l'aide d'un corpus de dessins réalisés par des élèves de collège en situation de lecture nous préciserons l'intérêt, mais aussi les limites, d'une méthode qui mobilise des outils de l'analyse documentaire pour établir une grille d'interprétation.

\section{Investir le dessin}

\subsection{Le dessin comme matériau d'investigation}

Depuis plusieurs siècles, les méthodes de recherches visuelles sont utilisées notamment en psychologie, en sociologie, en anthropologie, en archéologie (Knowles, Cole, 2008).

Le dessin de l'enfant est largement employé en psychologie dans l'objectif d'étudier le jeune dans ses activités. La représentation graphique est alors perçue comme la traduction d'une conception du monde par l'individu. La réalisation du dessin permet de récupérer des éléments sur le niveau ou la personnalité de son auteur. Le questionnement porte sur la correspondance de la perception de l'enfant avec celle de l'adulte ou sur la part du hasard dans la qualité du dessin (Baumstein-Heissier, 1955). Ce dernier est une source d'informations sur l'état de la représentation de l'image corporelle et sur le niveau de maturité du sujet. Dans son dessin, l'auteur projette une histoire vécue individuelle dans laquelle apparaît une maturité physique, mentale et affective. Il révèle une capacité d'entrer en contact avec son environnement (Nguyen, 1989).

En art thérapie, la méthode du portrait dessiné est aussi mobilisée. Il arrive que le patient se dessine par exemple avant et après une maladie ou un accident, révélant alors deux visages. Les dessins sont parfois associés à l'écriture de mots exprimant des sentiments : la souffrance, la fracture, le passé... (Delacheaume, Guillaume, 2016). Dans un autre cadre, un médecin psychiatre et deux animatrices ont organisé en 2009 une «activité portrait» pour des patients blessés majoritairement au niveau du visage (Sénéquier, Faleh, Jousselme, 2015). Cet atelier est perçu par les intervenants comme un atelier de remédiation corporelle. Ils souhaitent faire travailler l'image et la reconnaissance de soi après un traumatisme qui transforme le corps et sa représentation. Au fil des ateliers, la consigne évolue de : «dessinez-vous » à : «faites votre portrait tel que vous êtes aujourd'hui » avec l'introduction d'une temporalité. Les notions de ressemblance, d'acceptation de soi, de reconstruction sont dans ces domaines très fortement associées au portrait dessiné. L'art thérapie et plus précisément la réalisation de portraits permet d'obtenir des productions à travers 
lesquelles les analystes cherchent l'émergence de transferts d'émotions. Le climat créé lors des réalisations offre une verbalisation entraînée par le mouvement du dessin.

En sociologie de l'éducation, Constance Lavoie et Jo-Anni Joncas s'intéressent à l'éducation chez les Premiers peuples et chez les minorités ethnoculturelles. Elles présentent l'outil « dessinentretien » comme un instrument de collecte de données «élaboré afin de répondre aux lacunes méthodologiques liées aux recherches intégrant des communautés culturelles et linguistiques minoritaires » (Lavoie, Joncas, 2015). Cette méthode leur permet de travailler avec des communautés au-delà des difficultés liées au langage. Pour elles, il s'agit d'utiliser le dessin comme moyen de collecter et d'analyser des données dans le but d'atteindre une meilleure compréhension de l'objet étudié. Elles expliquent en quoi ce «dessin-entretien» fait ressortir des perceptions à travers des moyens non verbaux. Les dessins sont perçus comme des artefacts qui expriment des significations (Stock, Mares, Robinson, 2012). D'un côté les dessins-entretiens interviennent comme des facilitateurs de l'investigation, ils facilitent la collecte des données et le développement des connaissances concernant la population étudiée. D'un autre côté, ils autorisent la prise de parole et l'échange entre participants et chercheurs, ils simplifient le partage d'expériences et constituent un moyen pour mieux comprendre le vécu des participants (Lavoie, Joncas, 2015).

Ainsi, faire un dessin, c'est exposer un environnement connu, une reconstruction d'une réalité et une reconnaissance de soi, c'est renvoyer à une expérience vécue. A ce stade de notre exploration du sujet, produire un dessin apparaît comme réaliser un portrait au sens de reproduire une réalité et en interpréter les traits caractéristiques réels ou ressentis. Si les disciplines proches de la psychologie ont expérimenté dans leurs études l'intérêt du dessin et plus largement du portrait qu'en est-il en SIC ?

\subsection{Le portrait en Sciences de l'information et de la communication (SIC)}

En 1990, un groupe de chercheurs ${ }^{2}$ a réalisé une enquête qui lui a permis de dresser un profil sociodémographique des journalistes français (Service juridique et technique de l'information et de la communication, Commission de la carte d'identité des journalistes professionnels, Ministère de la culture et de la communication, Institut français de presse, 1991). Les auteurs ont analysé l'évolution d'une profession qu'ils montrent : rajeunie, féminisée, davantage diplômée, plus mobile mais aussi en crise d'identité. Cette enquête brosse les contours d'un collectif professionnel et offre une photographie, un portrait actualisé, qui se veut fidèle à ses changements.

Une autre recherche focalisée sur les liens qui se sont instaurées entre une population de chercheurs et une population de praticiens du domaine de l'information documentation a mis en lumière l'action d'un acteur majeur de leur rapprochement: Jean Meyriat. A la fois enseignant chercheur - Professeur à l'Institut d'études politiques, Directeur d'étude à l'école des hautes études et praticien de la documentation - Directeur du Centre de documentation de la Fondation nationale des sciences politiques - il a favorisé la production d'objets médiateurs (articles dans la revue Documentaliste-sciences de l'information ${ }^{3}$, édition de livres, informations sur l'actualité de la recherche, colloques et journées d'études) faisant intervenir des membres de ces deux populations. Analysés à partir de la théorie de la traduction élaborée par Michel Callon, les interactions qu'il a

\footnotetext{
${ }^{2}$ Il s'agit de : Valérie Devillard, Marie-Françoise Lafosse, Christine Leteinturier, Jean-Pierre Marhuenda, Rémy Rieffel, Claire Morin, Bel Kacem Mostefaoui et Isabelle Soing. Cités dans LAVILLE C. Les transformations de la pratique journalistique : le cas des correspondants étrangers de l'Agence France presse de 1945 à 2005. Thèse de doctorat, Faculté des études supérieures Université Laval Québec et Université de Paris VIII, 2007.

${ }^{3}$ Revue créée par l'ADBS (Association des documentalistes et bibliothécaires spécialisées) devenue l'association française des professionnels de l'information et de la documentation.
} 
construites ou permises entre ces deux populations pour assurer leur positionnement social en font un « acteur réseau », c'est-à-dire un intermédiaire agissant capable de relier deux mondes confrontés chacun à un problème. Les collaborations ainsi établies permettent de trouver des issues visant à résoudre le problème de l'un par la solution de celui de l'autre (Couzinet, 2000). Ce rôle tenu, observé à partir de ses prises de position, de ses engagements, de sa production de connaissance, de ses responsabilités institutionnelles ou associatives et qui prend en compte sa double appartenance sur l'ensemble de sa carrière, construit un ensemble de médiations hybrides. Ces dernières ouvrent une troisième voie aux recherches sur la communication entre chercheurs et la communication de vulgarisation et complètent l'étude du partage des savoirs en SIC (Couzinet, 2008a). L'analyse des actions mises en œuvre par Jean Meyriat dessine le portrait d'un chercheur, qui, impliqué au-delà de la sphère universitaire, est capable de mobiliser d'autres acteurs y compris dans la sphère professionnelle pour faire avancer la spécialité à laquelle il appartient l'information documentation.

Pour Adeline Wrona, le portrait est un genre journalistique, à partir duquel les journaux du XIX siècle donnent du sens au présent. Le portrait informe son lecteur par l'image. Il est un mode de connaissance de l'humain. A travers l'étude du Panthéon de Nadar (1852) et des albums Mariani (1894-1925), Adeline Wrona expose le développement d'un marché du portrait qui bénéficie des techniques modernes de reproduction des images. Ces portraits sont associés à des récits biographiques. Les journaux s'attribuent une mission de «publicisation » massive des visages d'une époque (Wrona, 2012).

Par ailleurs, Amanda Rueda (2014) a cherché à définir le portrait en SHS à travers le documentaire cinématographique. Elle explique comment le film interroge une part de vérité et d'objectivité dans la rencontre avec le sujet. Elle y intègre l'entretien car la parole constitue une matière essentielle. Pour elle, il permet d'aller à la rencontre d'un certain réel, à fabriquer un point de vue et une interprétation des singularités sur un portrait.

On voit comment les chercheurs en SIC dressent des portraits, soit pour aborder des actions ou enquêter pour déterminer une représentation, soit pour générer un profil ou pour traduire des comportements observés. Cependant, il s'agit surtout d'analyses de textes et d'entretiens et la forme graphique n'est que peu convoquée dans ces travaux, on peut alors se demander ce qu'apporte le dessin.

\subsection{Le dessin comme matériau empirique}

Le dessin en tant que matériau empirique s'inscrit dans une méthode qu'Isabelle Fabre a qualifiée de sensible (Fabre, 2018). Cette méthode permet de lire les espaces et les dispositifs en prenant en compte l'expérience qui s'y déroule et la manière dont les acteurs habitent les lieux. Cette approche s'apparente à une forme d'observation des pratiques et des interactions qui se déploient dans ces espaces ; c'est en particulier un support pour travailler sur l'analyse des pratiques professionnelles et sur l'analyse des usages des dispositifs. En relevant les traces de leur activité puis en analysant les discours qu'ils portent sur cette activité et le dispositif qui les entoure, on peut alors faire prendre conscience aux acteurs des différences de représentation et d'appropriation, mettre au jour des éléments qui contribuent à des déplacements ou à des masquages du projet initial d'un dispositif et qui peuvent inventer ainsi de nouvelles formes d'appropriation.

S'inspirant des travaux menés par Eliseo Véron (Véron, 1989, 1990) qui a travaillé d'une part sur l'espace d'une exposition et d'autre part sur les espaces de bibliothèques en dessinant les plans mettant en lumière les usages d'un lieu, cette méthode de recueil de données consiste à faire réaliser par les acteurs, un plan d'un espace soit conçu, soit vécu. Le dessin s'inscrit alors dans les méthodes visuelles qui sont utilisées pour déclencher la parole des interviewés lors d'entretiens individuels ou 
lors d'entretiens collectifs et faire surgir les représentations individuelles. Marie-Julie CatoirBrisson et Laura Jankeviciute ont utilisé ces méthodes visuelles dans leur approche des pratiques culturelles des étudiants et des usages numériques des préadolescents. Pour elles, il s'agit d'un procédé qui s'inscrit dans l'anthropologie de la communication car il est attentif au regard des usagers sur leur propre pratique. Elles s'interrogent pour savoir en quoi ces méthodes visuelles «peuvent être considérées comme un outil créatif de recherche qui renouvelle les techniques de l'entretien » (Catoir-Brisson, Jankeviciute, 2014).

Les supports d'entretien s'apparentent soit à des objets de la culture - des œuvres d'art, des extraits d'œuvres littéraires des planches de bandes dessinées par exemple (Fabre, 2006 ; Fabre, Couzinet, 2008) -, se nourrissant alors des artistes et de leur regard sur l'espace et le quotidien ; soit à des documents visuels créés par les acteurs eux-mêmes à partir d'une consigne (une photographie, un dessin, un plan par exemple), méthode utilisée dans des recherches concernant les espaces des centres de documentation et d'information de l'enseignement agricole (Fabre, Veyrac, 2008), de learning centre (Fabre, Gardiès, 2018) ou encore de learning lab (salle de pédagogie active) (Fabre, Gardiès, Fauré, 2019).

Une première phase d'analyse qui s'appuie, dans ces recherches, sur les images opératives (Ochanine, 1971) permet de constituer des ensembles avant de confronter les acteurs à ces représentations. En effet, le dessin comme trace est d'une part support d'entretien et d'autre part outil de confrontation des représentations mentales des concepteurs et des différents usagers de ces espaces et de leurs dispositifs. Même si, de premier abord, le passage par le dessin semble difficile pour la plupart des publics interrogés, il devient ensuite, à partir de l'étonnement qu'il suscite, un moyen d'amener les acteurs à se replonger dans leurs pratiques d'un espace et dans l'usage ou le non-usage des dispositifs. La représentation graphique facilite la parole, en particulier celle des jeunes, des élèves dans le cas des espaces documentaires du système scolaire. Lors de la réalisation du dessin du plan, l'élève se projette dans sa pratique de l'espace du Centre de documentation et d'information puis lors de l'entretien, la visite virtuelle suscitée par la confrontation au plan leur offre l'occasion de parler plus aisément de leurs usages de l'espace documentaire. Cette méthode rappelle les propos de Jean-Charles Lebahar : «la représentation graphique du monde réel et de ses objets, est une pratique de connaissance. Celle-ci consiste à interpréter ce monde en un langage spécialement conçu pour le transformer » (Lebahar, $1983: 5$ ).

Si les professionnels anticipent les besoins des usagers en termes d'utilisation des espaces, les dessins de plans ont permis une confrontation des regards. Les représentations graphiques mettent au jour des usages multiples et des vécus différents de l'espace documentaire. Elles sont une manière d'interroger à la fois l'espace conçu, l'espace perçu et l'espace vécu.

Le dessin utilisé en recherche constitue un réel apport puisqu'il permet d'exprimer graphiquement un ressenti, un vécu, un point de vue. Il peut être utilisé comme point de départ ou comme support pour réaliser un entretien et favoriser les échanges. Le portrait écrit ou dessiné est un genre qui tend à décrire tant au niveau moral que physique un individu, un groupe de personnes, une profession. Il incite celui qui le dresse à s'interroger sur les spécificités, les caractéristiques, le statut du portraituré. Serait-il envisageable de mener une recherche à partir de la méthode du portrait dessiné ? 


\section{Une méthode d'investigation : le portrait dessiné}

\subsection{Exploration documentaire}

Dans une première phase nous avons conduit une recherche d'information (RI) à partir des portails d'accès aux publications scientifiques Cairn, OpenEdition Journals anciennement Revue.org et Hal pour voir comment le dessin et le portrait sont abordés comme objet, méthode ou genre de recherche. Nous avons interrogé le champ résumé et le champ titre. Nous avons combiné les mots et expressions clés : «méthode », «portrait », « dessin », « méthode du dessin », « méthode du portrait », «méthode visuelle », « représentation graphique », et cet ensemble dans la discipline « science de l'information et de la communication » afin de recueillir les travaux qui s'y rapportent.

La RI à partir du mot « portrait » seul est celle qui donne le plus de résultats. Le genre du portrait se retrouve dans différents domaines : portrait sociologique, portrait de presse, portrait littéraire, portrait photographique, portrait historique, etc. Plusieurs revues à l'instar de Critique d'art ont une rubrique intitulée Portrait consacrée à la présentation du parcours de vie d'un personnage contemporain. On relève aussi la présentation du portrait d'une catégorie de personnes données renvoyant à une identité sociale : «Portrait de l'ethnographe en artiste » (Schmitt, Schmitt, 2016), «Portraits de terroristes » (Guibet-Lafaye, 2017), «Portraits de paysans » (Mauduit, 2015), des portraits sociologiques qui font écho aux travaux de Pierre Bourdieu et de Bernard Lahire (Bourdieu, Accardo, Balazs, Beaud, 1993 ; Lahire, Debroux, Denave, Faure, Glas, Millet, Renard, Tralongo, 2002). On note également des portraits de personnages historiques comme le « Portrait de Jésus séducteur » (Albert, 2000) ou « Le portrait de Che Guevara » (Maguet, 2010).

Dans une deuxième phase, parmi ces nombreux textes récupérés nous avons retenu des recherches portant à la fois sur le portrait comme genre à analyser et comme méthode d'investigation. Ainsi, l'introduction de l'ouvrage Pouvoirs du portrait sous les Habsbourg d'Espagne (Bodart, 2012) cherche à définir le portrait en y associant notamment les notions de ressemblance, de reconnaissance, de fonction mémorielle et d'identification. Le portrait fait la part belle au visage et plus particulièrement au regard. On trouve également des écrits abordant la question de ce qui fait un portrait photographique comme l'a fait Amanda Rueda pour le cinéma documentaire (2014). Par exemple, pour les anthropologues Jean Baptiste Eczet et Geremia Cometti, le portrait photographique s'impose comme un moyen efficace de représenter des personnes plastiquement, de mieux les approcher et d'apprendre à les connaître (Eczet, Cometti, 2017). Ces auteurs, souhaitent réaliser des portraits de migrants arrivés dans « la jungle de Calais ». Ce travail les pousse à définir le portrait photographique en anthropologie. Pour eux, il est l'association d'une description textuelle (ou récit de vie) et d'une description visuelle, sans qu'aucune des deux ne soit subordonnée à l'autre.

Martine Lavaud, chercheur en littérature, étudie les portraits photographiques d'écrivains (Lavaud, 2014). De son point de vue, ce qui prime dans le portrait photographique c'est le mécanisme de réception articulant l'œuvre et le portrait de l'écrivain. Elle explique comment le portrait de l'écrivain vient rencontrer son œuvre et comment il influence le public dans sa réception de l'œuvre littéraire de l'auteur photographié. Le portrait photographique apparaît comme un masque qui agit comme une «construction dont le texte [de l'écrivain] ne sort pas indemne ». Le portrait influence le public jusqu'à venir fabriquer la valeur littéraire d'un écrivain.

A travers ces travaux, le portrait est présenté avec des objectifs et des points de vue différents. Entendu comme méthode, il apparaît à la fois comme un moyen de production de connaissances d'une population donnée, une approche visuelle de soi ou d'un autre, une réflexion sur des manières d'être, sur des comportements, et une définition sociale d'un individu ou d'un groupe. A un autre 
niveau de réflexion, «portraits » au pluriel, permet de comparer plusieurs représentations afin de rapprocher, d'opposer, ou encore de suggérer des pratiques par exemple. Mener une recherche sur des adolescents lecteurs à partir de ces travaux nous paraît pertinent, tant cette méthode semble pouvoir nous conduire vers de nombreuses possibilités d'exploitation des données.

\subsection{La population dans son contexte}

A la recherche d'une méthode pour préciser l'intérêt du dessin, nous nous sommes intéressées au lecteur adolescent et avons retenu la représentation graphique utilisée par les psychologues et en SIC par Isabelle Fabre comme moyen de conscientiser une pratique. Nous sommes parties de l'idée selon laquelle un quasi consensus silencieux s'établit, en France, tant du côté de l'institution que du côté des familles, pour dire que la lecture est une pratique acquise au sortir de l'école primaire et n'a donc plus à être enseignée au-delà. Néanmoins afin d'en faire l'outil d'acquisition de savoirs disciplinaires, d'accès à l'autonomie dans la vie quotidienne et le moyen de formation tout au long de la vie, il nous paraît nécessaire d'amener à une prise de conscience des représentations qu'elle suscite. L'objectif poursuivi est de contribuer à apporter une aide apte à révéler les obstacles éventuellement rencontrés et à les dépasser. Nous nous sommes alors demandé quel rôle le professeur-documentaliste peut jouer dans cette prise de conscience des élèves de leurs propres pratiques et comportements de lecture. Qu'est-ce qu'un livre pour l'élève adolescent ? Comment se le représente-il ? Comment voit-il la lecture ? Comment la pratique-t-il ? Autrement dit, partant de l'hypothèse qu'ils ont tous en eux une représentation de la lecture et de leur rapport au livre, il s'agissait d'appréhender la manière dont les jeunes, au moment de l'entrée dans l'adolescence, pensent leurs pratiques de lecture.

Le terrain étudié est un collège des Pyrénées Orientales, situé en zone rurale. L'une d'entre nous, professeur-documentaliste dans cet établissement et donc en position observante, a recueilli les données empiriques qui ont servi de base de travail.

Pour comprendre les pratiques de lecture et les représentations des élèves, nous leur avons demandé de se dessiner puis de dessiner leurs camarades en train de lire. Il s'agissait de faire représenter leur perception du réel, des pratiques, des attitudes, des comportements, des rapports entretenus avec le livre. Trois points de vue ont été retenus. Le premier est le regard sur soi révélé d'une part, par l'autoreprésentation graphique ce qui a permis d'obtenir un corpus de dessins, et d'autre part, par le discours d'autres jeunes au travers de bribes de récit de soi, qui expriment des expériences de lecture. Ces verbatims complètent ou viennent en écho aux dessins. Le deuxième point de vue est le regard des élèves sur leurs pairs en train de lire. Enfin, le troisième est le regard du professeur-documentaliste, observateur des élèves par l'intermédiaire de dessins. Un nouveau corpus a ainsi permis de saisir des attitudes et d'opérer des croisements avec les dessins réalisés par les élèves eux-mêmes. Afin de pouvoir recueillir des données suffisamment significatives et valides, l'élaboration de ces trois points de vue tient compte du contexte particulier de l'enquête.

\subsection{La consigne et les dessins obtenus}

Deux types de travaux ${ }^{4}$ ont permis de faire réaliser des dessins à des élèves en fin de classes de CM2 et de classes de cinquième. D'autres dessins ont été recueillis, de manière isolée, d'élèves de quatrième et de troisième, mais ils n'ont pas été assez nombreux pour offrir des observations

\footnotetext{
${ }^{4}$ Le premier travail est conduit par le professeur-documentaliste auprès d'élèves de CM2 du secteur avant leur entrée au collège. Il a lieu lors de la journée de visite de l'établissement, au mois de juin. Le second travail est réalisé dans le cadre d'un partenariat entre le professeur-documentaliste et un enseignant d'arts plastiques. Dans les deux cas de figure, les élèves réalisent leur production au CDI. 
significatives. En outre, nous avons constaté deux attitudes opposées : soit le refus de dessiner, car cette activité est, aux yeux de certains, réservée aux plus jeunes ; soit au contraire un engagement dans le dessin qui se révèle par des traits ou des choix plus affirmés.

La consigne formulée par l'enseignant s'insère dans un processus d'apprentissage, elle renvoie à une «vérification de connaissances, ou à une recherche, ou à une concrétisation de savoirs enseignés sous la forme d'une production »(Zakhartchouk, 1996). Elle est la condition de réussite d'un travail et doit permettre à l'élève de s'approprier ce qui lui est demandé. Ainsi lorsque la formulation de notre consigne n'était pas assez précise ou mal définie, l'élève n'arrivait pas à se projeter mentalement et à réaliser son dessin. Nous avons cherché progressivement à le conduire verbalement vers un cheminement réflexif et procédural de sa propre pratique de lecture, à se remémorer des actions passées souvent effectuées de manière non réfléchie. Ainsi accompagné, il parvient à conscientiser son attitude, à se remémorer une posture prise plus souvent qu'une autre, à se concentrer sur des gestes faits souvent de manière instinctive. C'est en le guidant de la sorte que le travail de représentation de soi devient efficient et qu'on peut commencer à le voir au travers des dessins.

Sur un total de cent quatre-vingt-dix-huit dessins d'élèves recueillis, seuls seize ont été retenus pour la constitution de notre corpus. Plusieurs dessins ont été écartés du corpus pour des raisons différentes. Dans un premier temps, nous avons écarté les dessins pour lesquels nous n'avions pas obtenu les autorisations des représentants légaux ${ }^{5}$. Dans un deuxième temps, nous avons refusé les dessins non réalisés avec sérieux, les copies blanches ou les esquisses. Dans un troisième temps, nous avons exclu les dessins qui se ressemblaient, soit parce que les jeunes se sont copiés durant l'exercice, soit parce que nos consignes étaient trop précises et influençaient les jeunes dans leurs choix de représentation. Ces dessins montraient de manière ostensible des mises en scènes trop similaires. Dans un quatrième temps, deux autres types de dessins ont été écartés, mais pourraient faire l'objet d'un prolongement à la recherche présentée ici. Ce sont ceux qui ne présentent pas de livre ou ce qui font appel à l'imaginaire comme le dessin d'un héros par exemple.

Le choix définitif s'est porté sur l'unité et l'homogénéité et l'interprétabilité. Le critère d'unité et d'homogénéité nécessite dans notre cas que les sujets aient le même niveau d'étude afin de pouvoir comparer les dessins. L'interprétabilité est un critère de qualité déterminant pour un corpus (Pincemin, 2007). Il appelle la question de la traduction, de la transcription, de la translation du visuel au verbal et celle des contextes et des relations.

Le dessin peut-il être un moyen de recueillir des données ? L'ensemble des dessins constitue une production singulière que l'on peut qualifier de documentaire en ce qu'elle porte des éléments signifiants. Il montre des jeunes lisant, il révèle des expériences intimes. L'analyse du contenu conduit à élaborer une grille apte à en révéler la productivité de l'ensemble comme moyen d'investigation et à produire des interprétations.

\footnotetext{
${ }^{5}$ Les dessins recueillis sont considérés comme des travaux pédagogiques réalisés dans le cadre scolaire. Ils sont des œuvres originales car ils reflètent la personnalité de leurs auteurs. Quels que soit leur genre, leur forme d'expression, leur mérite, leur destination et leur support, ils sont protégés par le Code de la propriété intellectuelle (Article L112-1). Ils sont donc protégés par le droit d'auteur. Les élèves interrogés étant mineurs, nous avons demandé l'autorisation de leurs responsables légaux pour pouvoir utiliser ou diffuser les dessins.
} 


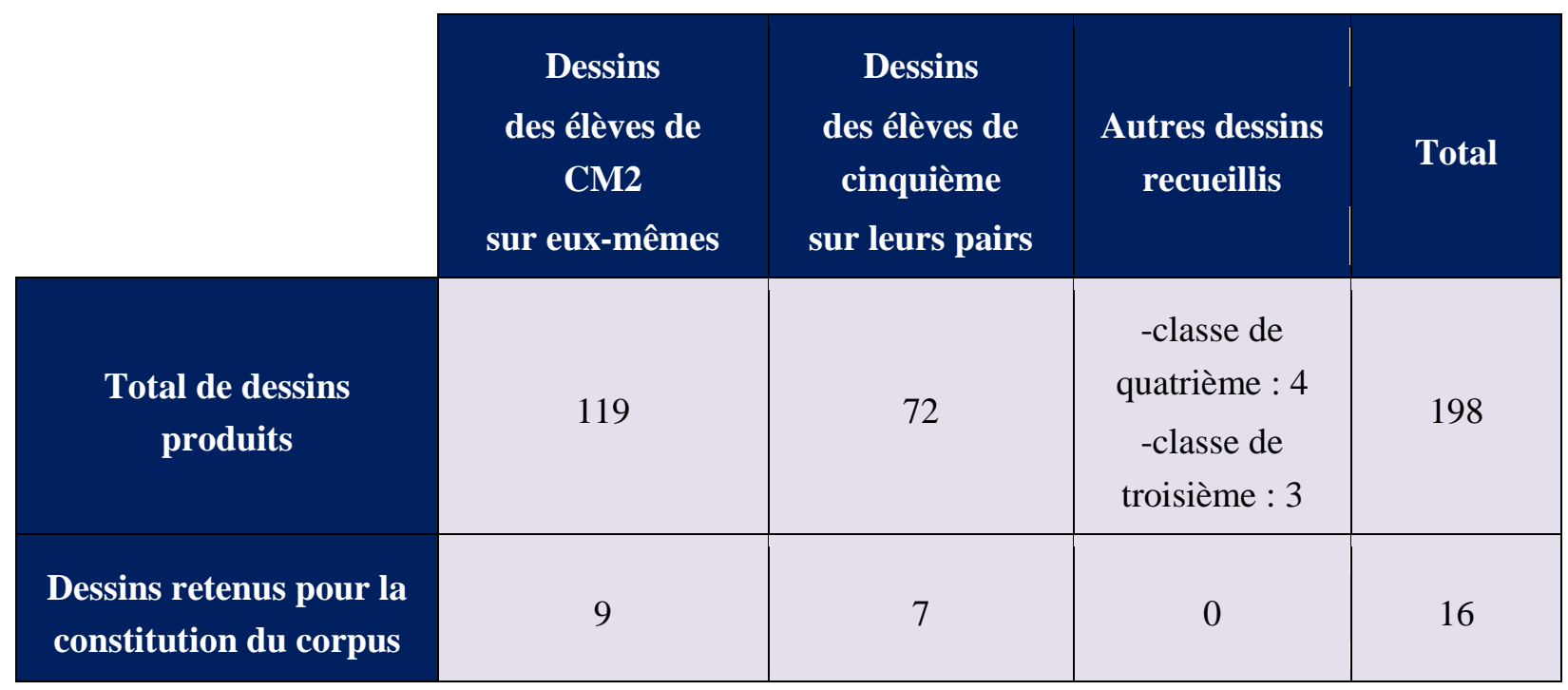

Tableau 1. Recueil des dessins d'élèves et choix opérés

\section{Le dessin comme méthode d'analyse : du portrait à la scène}

\subsection{Le recours à un langage documentaire}

Une grille interprétative se fonde sur un ensemble d'éléments lexicaux permettant de faire correspondre une observation à une verbalisation imprégnée de la situation dans laquelle elle se trouve. Les pratiques de lecture que nous cherchons à observer s'insèrent dans les pratiques documentaires. Elles sont des manières d'appréhender un document c'est-à-dire d'être capable de le lire, de le comprendre et d'en tirer des informations transformables en connaissances. Il s'agit donc d'une attitude active qui nécessite des acquis préalables, comme savoir lire, savoir comprendre le sens d'une grande partie du vocabulaire, savoir se concentrer, savoir organiser et exploiter des idées et savoir s'abandonner au plaisir de la découverte ou de l'évasion.

En information-documentation on manipule et on fabrique des outils, les langages documentaires, permettant d'analyser des contenus. Ces analyses, dont les résultats s'expriment en codes ou en mots et expressions sont aussi des interprétations. Il nous a semblé utile de nous référer à ce type d'outil pour préciser un vocabulaire interprétatif du contenu et élaborer ainsi une grille afin d'analyser une production documentaire.

Le langage documentaire est un langage artificiel permettant de représenter le contenu des documents. Il sert à la fois à garder une trace du document et à le retrouver à partir de l'information qu'il contient. Il est un intermédiaire entre les documents et les besoins d'information des usagers. «La fonction première d'un langage documentaire est d'ordonner, donc de classer c'est à dire de placer des objets documentaires les uns par rapport aux autres, dans un ensemble, en prenant en compte une caractéristique particulière. L'objectif est donc, à la fois, de rassembler et de différencier au sein de sous-ensembles distincts ॥ (Couzinet, 2008). C'est cette capacité à différencier des objets documentaires, dans notre cas les textes des verbatims et les dessins, qui nous intéresse ici.

Afin d'élaborer notre grille interprétative nous avons retenu cinq outils qui ont permis, à partir de leur vision du lecteur et de la lecture, de sélectionner du vocabulaire. Les terminologies employées nous permettent de comprendre et de décrire l'acte de lire. Notre choix s'est porté sur des outils destinés à indexer des représentations graphiques ou destinés à indexer des objets documentaires 
visant à promouvoir la lecture auprès de populations particulières, ou encore à indexer des objets portant sur le livre et la lecture.

Le thésaurus iconographique Iconclass $^{6}$ créé aux Pays-Bas dans les années 1950 par le professeur en histoire de l'art Henri Van de Wall (1910-1972) est traduit dans plusieurs langues et enrichi au fil des ans. Cet outil est utilisé par les musées et les institutions au niveau international pour décrire les œuvres d'art, les livres illustrés, les reproductions, les photographies et les diapositives. Un extrait de ce thésaurus présente le concept de lecture de la manière suivante : 49N1 : faire la lecture à quelqu'un ; 49N2 : accessoires de lecture ; 49N21 pupitre, lutrin ; 49N22 baguette servant à indiquer le texte - lecture ; 49N23 signet de livre.

L'Unesco, une institution de l'Organisation des Nations Unies, est spécialisé dans les domaines de l'éducation, la science et la culture. Son thésaurus ${ }^{7}$, dont la première version a été établie en 1974, donne au concept de «Lecture » un terme préférentiel : « Habitude de lecture ». A ce concept, deux autres sont associés : «Enseignement de la lecture » et «Promotion de la lecture ».

Au moment de la conception du Thésaurus iconographique en 1984, François Garnier a cherché à pouvoir indexer toutes les formes d'expressions figurées, aussi bien les peintures rupestres que des toiles, des estampes, des vitraux, des plans architecturaux ou des images de publicité. La banque de données Joconde, portail des collections des Musées de France, disponible sur internet depuis 1995, utilise ce langage documentaire. Dans ce thésaurus, François Garnier expose trois formes de représentations humaines : les figures, les portraits et les scènes.

Le quatrième outil est le site internet Getty Images $^{9}$, une banque d'images et de vidéos américaine développée au départ à partir de photos d'actualité, de sport ou de divertissement. Des millions de photographies sont indexées par les usagers eux-mêmes. Tout utilisateur peut participer à la description d'un document. Les mots clés employés sont nombreux et mêlent niveaux généraux et spécifiques et décrivent à la fois forme et contenu de l'image (Régimbeau, 2001). A partir du motclé «lire » et des filtres, type d'image : illustration et style d'image : portrait, les images sont définies par des termes très différents. Nous relevons : des lieux, des espaces-temps, un nombre de personnes, des liens entre les personnes représentées, des parties de corps humain, des gestes ou des actions, des particularités physiques, des traits comportementaux, des éléments de décor, le sujet du livre lu.

Les derniers outils sont les schémas de classification de la bibliologie réalisés par l'un des précurseurs de la bibliologie, Al-KalKashandi (1355-1418) ${ }^{10}$. Ils nous indiquent des traces de l'importance des gestes dans la formation du Katib. Cette présentation, qui date du $\mathrm{XV}^{\mathrm{e}}$ siècle, est détaillée avec clarté et révèle l'importance déjà accordée à cette fonction. Le Katib ou le secrétaire, dans la culture arabo-musulmane, désigne la personne en charge de rédiger les documents officiels : il est le secrétaire de la chancellerie. Il n'est ni écrivain, ni copiste. Du fait de ses missions, il occupe un haut rang dans la hiérarchie sociale de l'époque. Al KalKashandi a réalisé ce schéma de classification de la bibliologie dans le but de former des secrétaires dans le métier de la rédaction. Son travail montre une vision de la discipline bibliologie et une approche intéressante par l'écriture et l'écrivain (Couzinet, 2008b). Il énonce à travers son travail de taxinomie trois ensembles : 1. l'écriture et l'écrivain, 2. La formation théorique du Katib, et 3. La formation pratique du Katib. Dans ce troisième ensemble, Al KalKashandi propose une partie consacrée à la physiologie de

\footnotetext{
${ }^{6}$ ICONCLASS. Disponible sur : http://www.iconclass.org/help/outline Consulté le 11 juin 2019.

${ }^{7}$ UNESCO. Disponible sur : http://vocabularies.unesco.org/browser/thesaurus/fr/ Consulté le 11 juin 2019.

${ }^{8}$ GARNIER F., Thésaurus iconographique : système descriptif des représentations, Le Léopard d'or, Paris, 1984.

${ }^{9}$ GETTYIMAGES. Disponible sur : http://www.gettyimages.fr Consulté le 11 juin 2019.

${ }^{10}$ GDOURA W., «La conception de la bibliologie chez Al-Kalkashandi (VXe siècle) », dans Actes du $9^{e}$ colloque de bibliologie (Tunis, 20-24 mars 1990), Tunis, IPSI, 1993. 
l'écriture où apparaissent des gestes propres à l'exercice de la fonction du Katib : la manière de tenir le calame, la manière d'alimenter la plume en encre, la manière de mettre la plume sur l'oreille au moment où le Katib réfléchit. Dans les règles d'écriture, on trouve aussi : le mouvement de la main au moment de l'écriture et l'inscription des caractères (Rebhi, Odoura, 1990).

Ces listes thématiques, alphabétiques, hiérarchiques, regroupent des mots-clés et des descripteurs qui constituent des points d'ancrage conceptuels. La structure des langages documentaires explorés ont mis en évidence des degrés de profondeur, une position des concepts dans une arborescence, des équivalents dans d'autres langues, des styles d'écriture, des dits et des non-dits.

Evoquer le rôle communicationnel des langages documentaires c'est s'interroger sur le rôle intrinsèque de la documentation, à savoir la manière dont le praticien catégorise l'information et choisit de la représenter. Pour Jean Meyriat, l'approche sémiologique entre dans la spécificité des études info-communicationnelles (Meyriat, 1993). Le traitement des contenus informationnels opéré par les praticiens de la documentation est en effet un travail sur le sens. Les langages documentaires donnent non seulement un accès facilité aux images mais ils aident aussi à déchiffrer les informations grâce à des critères descriptifs empruntés à l'analyse de contenu (Bardin, 2013). Le vocabulaire constitué de descripteurs, mots ou expression sélectionnés pour le constituer en thésaurus, porte en lui une charge informationnelle. Pour El-Khansa Mkada (2012) il est un acte de discours. Dans sa conception du thésaurus, cet auteur considère le descripteur comme un acte fort d'énonciation. Il porte en lui une intention manifeste de dire quelque chose. Les concepteurs du langage documentaire par les choix qu'ils opèrent, comme les praticiens qui emploient tel ou tel énoncé, participent à la diffusion d'un discours.

Ils sont des outils d'observation et de compréhension d'un environnement. De nombreux travaux en SIC ont mis en évidence ce rôle particulier. Dans le contexte de l'enseignement du second degré par exemple il permet à l'élève de comprendre le sens de termes qu'il ne connaît pas en fonction de la position de ce terme dans une hiérarchie (Couzinet, 2011). Ils ont été également analysés afin de mettre au jour comment une discipline, en l'occurrence l'information-documentation, est perçue dans la société (Couzinet, 2008b ; Couzinet, 2009) ou comment ils portent et transmettent des stéréotypes (Courbières, 2010). De plus en plus spécialisés, ils participent également par le contour d'un domaine qu'ils tracent à le positionner par rapport à d'autres. C'est ainsi qu'ils ont pu être utilisés pour porter le projet des SIC comme discipline auprès des pouvoirs publics (Meyriat, 1983) et pour délimiter la bibliologie (Estivals, 1993; Saldanha, 2019) comme science du livre et de l'écrit. Les langages documentaires révèlent alors des représentations que des recherches qualitatives mettent en lumière (Couzinet, 2016). Ils peuvent donc être utilisés pour établir des grilles servant à interpréter des contenus de documents.

Les dessins produits par les élèves sont des objets documentaires car ils sont porteurs, comme l'ont montré les psychologues et les travaux d'Isabelle Fabre, d'informations sur la manière dont ce qui est représenté est perçu. Les langages documentaires sélectionnés constituent une base de vocabulaire qu'il est possible de compléter pour interpréter cette production des élèves. Pour la recherche présentée ici nous nous limitons à montrer en quoi le dessin peut contribuer à l'interprétation.

\subsection{Le livre et la lecture à travers le dessin}

Les dessins ${ }^{11}$ témoignent du regard que les élèves portent sur la matérialité des supports du livre. Ce dernier est envisagé comme un objet structuré en deux parties symétriques. On le reconnaît

\footnotetext{
${ }^{11}$ Les titres des dessins sont donnés par les élèves, les prénoms des élèves ont été changés pour préserver l'anonymat. 
notamment à travers l'épaisseur des ouvrages et leur ossature (dessin 1): une première de couverture titrée, un dos et une quatrième de couverture.

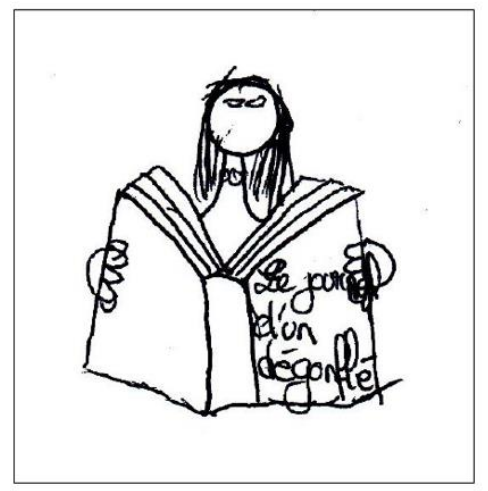

Dessin 1. Etre dans le livre, Mathilde, 11 ans (Point de vue d'un élève sur lui-même)

Bien que non représentée par les élèves, on note sur l'un des dessins de l'observatrice l'utilisation d'une liseuse ${ }^{12}$. C'est un choix délibéré de représenter ce support (dessin 2). Il découle de l'observation. Si les élèves n'ont pas choisi de se représenter avec une liseuse mais ont préféré le support imprimé on peut y voir que le support numérique n'est pas encore inscrit dans leurs pratiques de lecture et que, pour eux, le livre est un objet qui a une existence matérielle propre. Pourtant, quelques-uns, très peu nombreux, l'utilisent au collège.

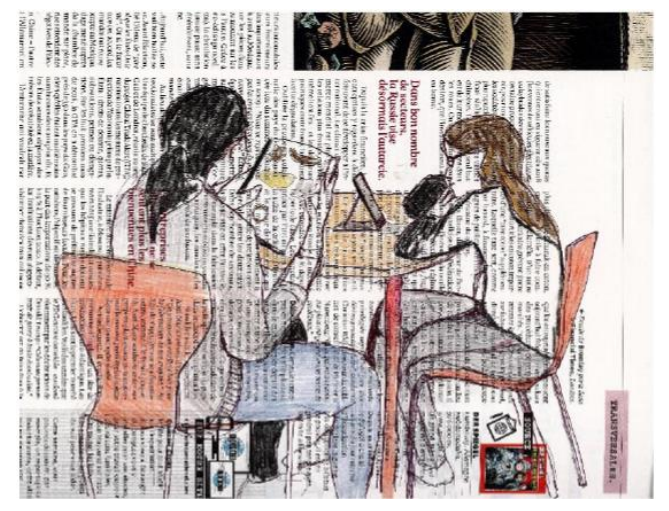

Dessin 2. Des supports de lecture différents, Observateur (Point de vue du professeur-documentaliste en situation d'observation)

Sur les dessins apparaissent des contenus à travers des titres, des lignes ondulées représentant le texte, des cases représentant des images (dessin 3).

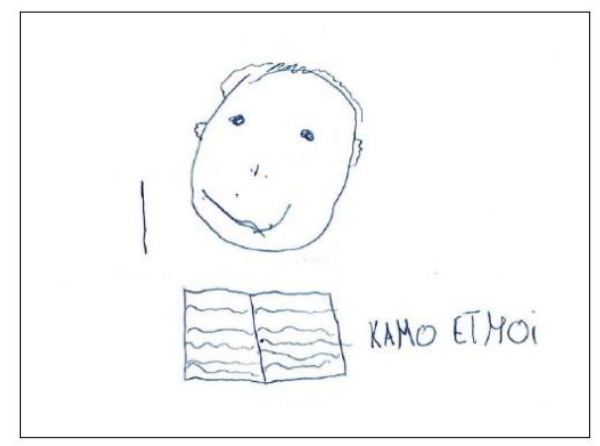

Dessin 3. La tête et le livre, Quillian, 11 ans (Point de vue d'un élève sur lui-même)

\footnotetext{
${ }^{12}$ Le Centre de Documentation et d'Information dispose de deux liseuses pour le prêt aux élèves. Dans le cas du dessin, la liseuse appartient à l'élève.
} 
Si certains laissent voir des contenus et une matérialité forte, d'autres proposent des livres de pages blanches. Bien qu'eux aussi ouverts sur deux pages en vis-à-vis ils n'offrent aucun indice quant à leur contenu (dessin 4), on peut alors se demander s'il s'agit de représenter une chose insignifiante ou que l'on veut garder secrète au regard intrusif de l'observateur ou des pairs (dessin 7).

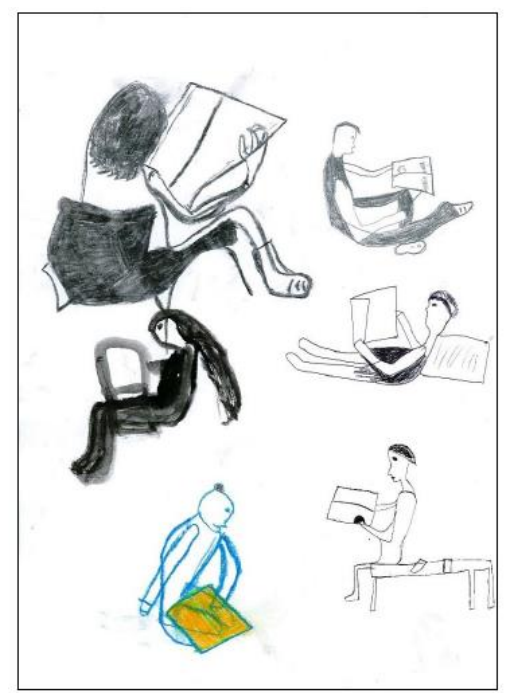

Dessin 4. Des livres blancs, Sacha, 12 ans (Point de vue d'un élève sur ses pairs)

L'environnement de lecture qu'ils montrent révèle les différents rôles que jouent le livre et la lecture. Lire pour s'informer, pour s'endormir, faire une pause, s'évader, s'isoler, partager. Ceci est renforcé par les différents espaces privilégiés, reconnaissables notamment au travers du mobilier. Ce peut être le Centre de documentation et d'information, lieu de recherches documentaires et espace de lecture. D'autres espaces du collège permettent de voir des lectures à plusieurs, des lectures côte à côte, dans les escaliers ou dans la salle polyvalente, salle importante par sa capacité d'accueil et par sa diversification d'activités. A la fois salle de réunion, salle d'exposition, salle audio-visuelle, salle de spectacle, elle accueille de multiples manifestations qui peuvent être culturelles, d'initiation aux premiers secours... Dans un environnement plus intime ce peut-être une chambre, un moment d'isolement plus favorable à la concentration ou à l'évasion (dessin 5).

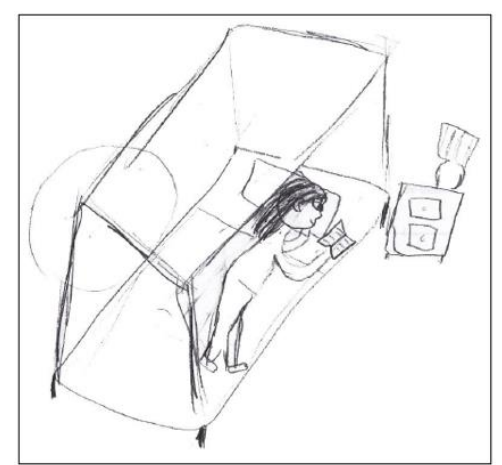

Dessin 5. Ma chambre, mon livre et moi, Manon, 10 ans (Point de vue d'un élève sur lui-même)

Nous observons également des manières de lire qui transparaissent au travers des postures corporelles favorables ou non dans un contexte de lecture plaisir ou de lecture contrainte prises par les lecteurs. Plusieurs sont représentés de dos comme si le moment n'appartenait qu'à eux (dessin 6 et 7). On note aussi des façons de se saisir du livre : des deux mains tel qu'on le ferait dans une confrontation corps à corps ou pour marquer l'appartenance ; du bout du vêtement parfois, il peut 
alors s'agir d'un objet perçu à peine touchable et dont il faut se protéger du contact physique (dessin $6)$.

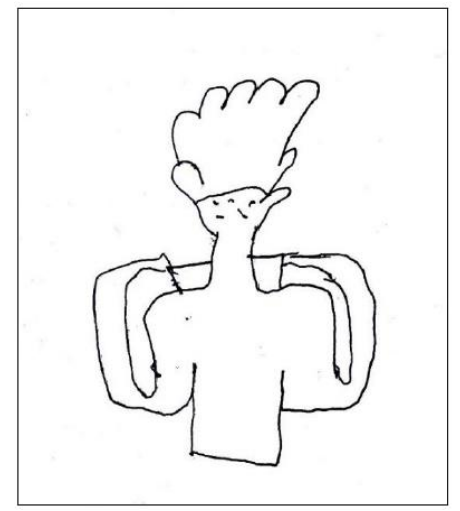

Dessin 6. Lire le dos tourné, Mathis, 12 ans (Point de vue d'un élève sur lui-même)

Des ressentis sont perceptibles sur les visages des lecteurs. Ils sont visibles au travers des expressions faciales, des regards dévoilant une intériorité, une concentration, le plaisir ou la souffrance, voire l'indifférence (dessin 7).

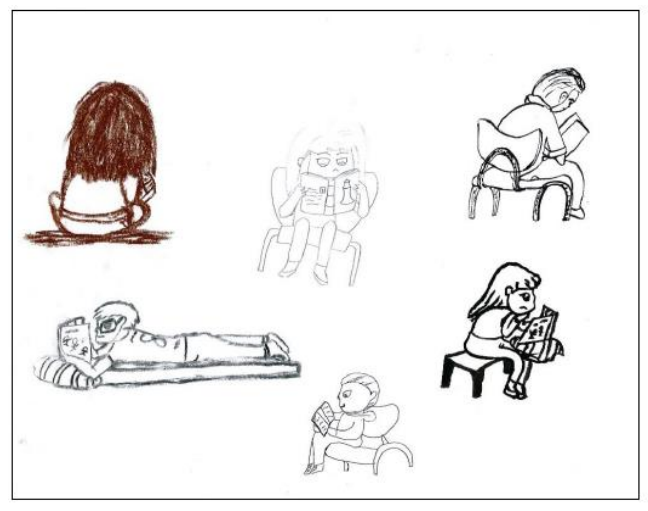

Dessin 7. Positions et attitudes variées, Carla, 12 ans (Point de vue d'un élève sur ses pairs)

La lecture peut être vécue comme un moment partagé. Les lecteurs éprouvent, de manière plus ou moins consciente, un sentiment d'appartenance à un groupe dans lequel les individus s'engagent, s'impliquent, participent. D'une certaine manière, la lecture les lie. Cette manière de lire ensemble les fait appartenir à une communauté de lecteurs. Ces-derniers ont des pratiques communes, des gestes qui se ressemblent, ils forment une «communauté de pratique », expression empruntée à Karel Soumagnac-Colin (2016), (dessin 8 et 9).

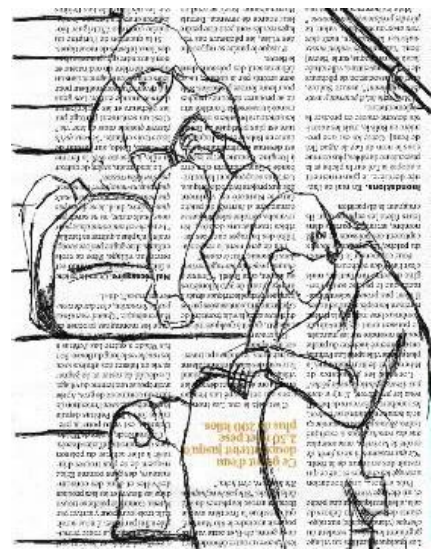

Dessin 8. Lecture dans l'escalier, Observateur (Point de vue du professeur-documentaliste en situation d'observation) 


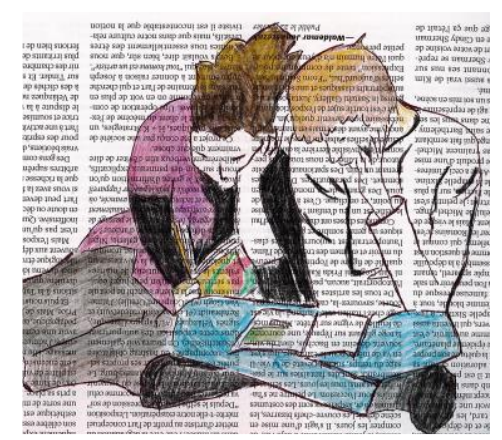

Dessin 9. Lire côte à côte, Observateur (Point de vue du professeur-documentaliste en situation d'observation)

Au sein de cette communauté de lecteurs certains sont plus à l'aise que d'autres, ils ont leurs habitudes, arrivent aisément à entrer dans une bulle qui leur permet de s'isoler, de se retirer du réel. Leurs attitudes et leurs comportements peuvent constituer un modèle pour d'autres. Les gestes qu'ils effectuent sont comme des codes qu'ils transmettent à leur entourage, comme peuvent le faire des parents pour montrer l'exemple à leurs enfants ou un maitre à ses élèves (dessin 10).

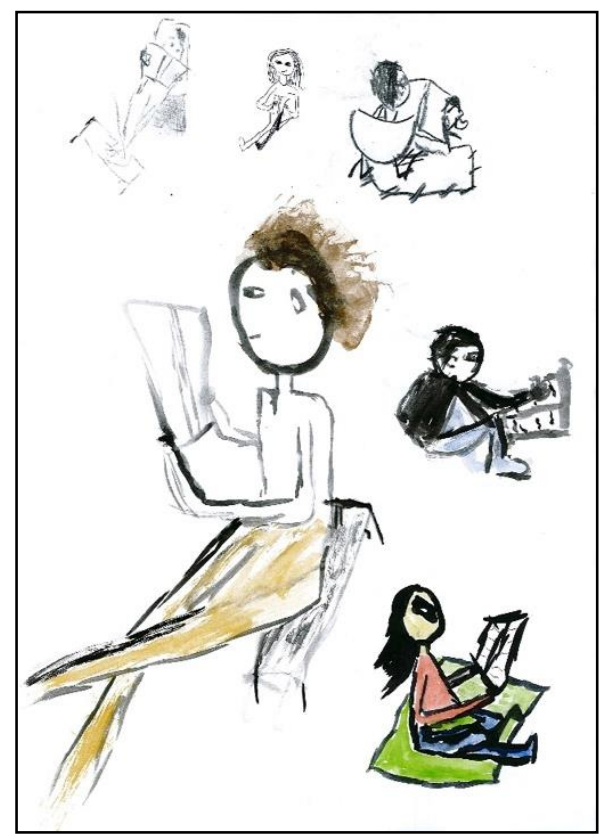

Dessin 10. Maman montre l'exemple, Emilie, 12 ans (Point de vue d'un élève sur ses pairs)

Si le moment de lecture et l'ambiance dessinée est souvent représentée de manière claire avec un lecteur tenant un support de lecture dans les mains et/ou positionné de façon à avoir le regard orienté vers le texte, il arrive que certains dessins représentent une activité plus confuse dans laquelle l'individu ne lit pas. Ce temps de non lecture peut être choisi comme pour méditer sur ce qui vient d'être lu, à moins que l'auteur du dessin choisisse délibérément de représenter un non lecteur. Le dessin 11 laisse penser que les personnes représentées n'arrivent pas à lire, en effet, nous avons du mal à reconnaître des personnes lisant tant le dessin est instable, désordonné, chaotique, comme si l'ordre, la stabilité, le calme était nécessaire à la lecture (dessin 12). 


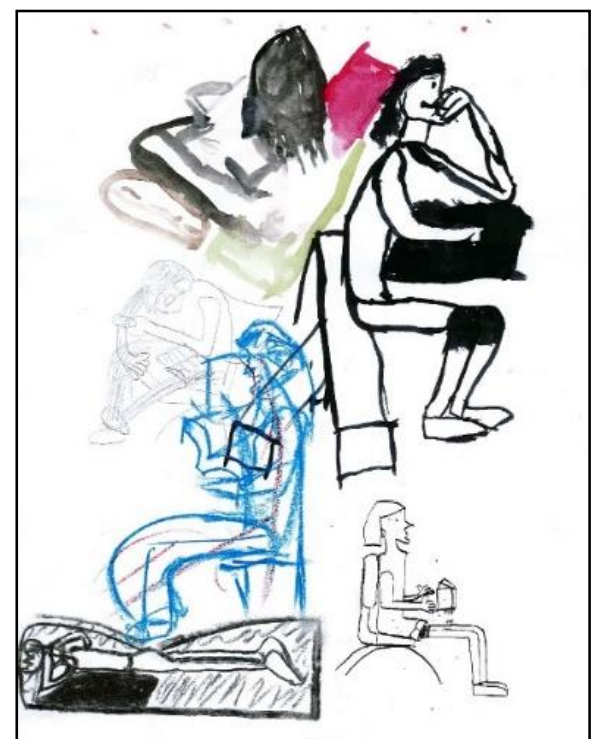

Dessin 11. Trois livres pour six lecteurs ou L'inverse de lire, Arthur, 12 ans (Point de vue d'un élève sur ses pairs)

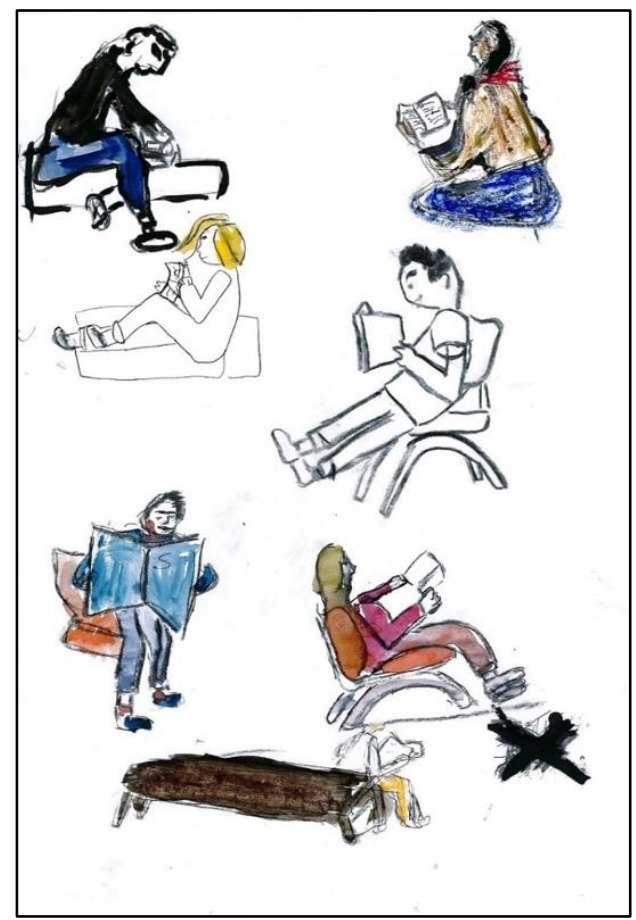

Dessin 12. Lire au CDI, Iris, 12 ans (Point de vue d'un élève sur ses pairs)

\subsection{Interpréter les dessins : du portrait à la scène}

Ces relevés fourmillent de gestes et d'actions des sujets représentés. Si au départ nous souhaitions utiliser la formulation de «portrait», la poursuite de ce projet interroge la qualification même des dessins. L'interprétation guidée par la grille permet alors de qualifier le type de dessin auquel on a affaire. C'est le passage et l'examen des langages documentaires et notamment le recours au Thésaurus iconographique de François Garnier qui nous a conduit à opter pour la sous-catégorie de la «scène de genre : scène de lecture », entendue pour Ségolène Bergeon Langle et Pierre Curie comme la «représentation de l'action anecdotique d'un ou de plusieurs personnages » (Bergeon Langle, Curie, 2009). Selon François Garnier, le portrait, contrairement à la scène, renvoie à un personnage n'exécutant aucune activité (Garnier, 1984). En ce sens, Jean-Luc Nancy précise que 
« le portrait véritable est donc bien concentré dans ce que les historiens de l'art ont désigné sous la catégorie de «portrait autonome », celui dans lequel le personnage représenté n'est pris dans aucune action ni même ne supporte aucune expression qui détourne de sa personne elle-même » (Nancy, 2000 : 15). On peut dire que le portrait écarte l'action du sujet représenté. C'est donc bien des scènes de lecture et non des portraits que les élèves, sans le savoir, ont dessiné. Ils se sont mis en scène à travers une série d'actions.

Il est vraisemblable que chemin faisant dans l'analyse la requalification du «portrait» en « scènes », imperceptible dans la revue de la littérature que nous avons dressée (partie1) aurait été faite. Néanmoins ici le langage documentaire fait gagner un temps précieux. Il amène aussi à modifier la grille de lecture. Celle-ci ne se limite plus à des objets. Elle contient des éléments du rapport corps/livre.

\begin{tabular}{|ll|}
\hline Rapport corps / livre & \\
& Corps caché par le livre \\
& Corps confondu avec le livre \\
& Corps déconnecté du livre \\
& Corps gêné par le livre \\
& Livre caché par le corps \\
& Livre posé devant soi \\
& Livre posé sur les jambes \\
& Livre posé sur les cuisses \\
& Livre posé sur le siège \\
& Visage près du livre \\
\hline
\end{tabular}

Tableau 2. Extrait de la grille d'analyse et d'interprétation sur le rapport corps livre

Elle contient également des actes. Ceci permet de percevoir la relation que celui qui se dessine établit avec l'objet et facilite l'interprétation.

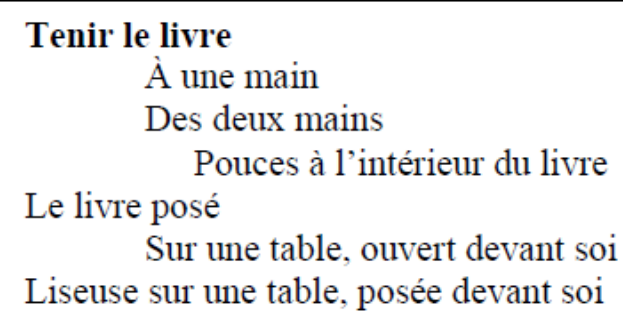

Tableau 3. Extrait de la grille d'analyse et d'interprétation sur tenir le livre

La grille interprétative peut être complétée par des éléments de description de l'environnement ici mobilier ou amical. Les amis ou partenaires de lecture participent à la scénographie qu'ils enrichissent par la contextualisation qu'ils proposent. L'introduction de «scène » suggérée par le Thésaurus Garnier ${ }^{13}$ est une qualification qui nous semble révéler les pratiques.

Ainsi le couplage dessin-outil documentaire autorise des interprétations de représentation du contenu en évitant l'écueil d'analyses psychologiques qui ne seraient pas fondées théoriquement et qui ne relèvent pas des SIC. Enfin, le croisement des représentations graphiques effectuées par les pairs ou les observateurs nuancent les interprétations en proposant des regards différents ou partagés en fonction de la place de chacun.

\footnotetext{
${ }^{13}$ Pour Garnier, « le terme scène a un sens très général. Il recouvre toutes les compositions dans lesquelles l'être humain ou divin, seul ou non, est représenté en action : paysan taillant la vigne, homme en prière, dispute de vieillards... » Garnier distingue dans son thésaurus les scènes bibliques, les scènes historiques et les scènes mythologiques (Garnier, 1984, pp. 40-49).
} 


\section{Conclusion}

Introduire l'expression graphique comme matériau empirique contribue à révéler les représentations. Dans cette recherche elle est la figuration d'une scène graphique brute, produite par les élèves, et le dessin d'une scène graphique interprétée par l'observateur.

Le recours aux outils d'analyse des professionnels de l'information-documentation montre que l'apport du dessin peut être considérable tant sur le plan de l'observation de pratiques que sur le plan de la définition des objets observés. Dégager de ces outils des concepts descripteurs permet de nommer le visible. Dans notre étude il s'agit de supports, de comportements, d'attitudes et de relations. On peut dire que la méthode visuelle telle que nous l'avons décrite, associée à l'apport des langages documentaires permet d'accorder visible et dicible.

Le dessin est encore faiblement utilisé comme méthode d'investigation en SIC. Nous avons conscience que, comme dans toute recherche il ne peut à lui seul tout révéler. La confrontation à une analyse de documents ou à l'expression verbale pour développer, confirmer, nuancer, opposer voire contredire et aussi interroger les situations observées nous semble nécessaire. Comme image brute et isolée il est peu exploitable, mais resitué dans un contexte, il est un moyen d'exprimer des représentations dans un environnement.

\section{Bibliographie}

Albert J.-P., «Portrait de Jésus en séducteur », Les Cahiers du Centre de Recherches Historiques, vol. 24, 2000. doi : $10.4000 /$ ccrh. 1942 .

BARDIN L., L'analyse de contenu, Presses Universitaires de France, Paris, 2013.

BAUMSTEIN-HEISSLER N., «A propos du dessin: quelques opinions et travaux de psychologues soviétiques », Enfance, tome 8, n 4, p. 377-399, 1955.

Bergeon langle S., Curie P., Peinture \& dessin. Tome 1. Vocabulaire typologique et technique, Editions du patrimoine, Centre des monuments nationaux, Paris, 2009.

Bodart D. H., Introduction: Dialogue avec le portrait, in Pouvoirs du portrait sous les Habsbourg d'Espagne, CTHS : INHA, Institut national d'histoire de l'art, Paris, 2012.

BouRdieu P., ACCARDO A., BALAZS G., BEAUd S., La misère du monde, Seuil, Paris, 1993.

CATOIR-BRISSON M.-J., Jankeviciute L., «Entretien et méthodes visuelles : une démarche de recherche créative en sciences de l'information et de la communication », Sciences de la société, nº 92, p. 111-127, 2014.

COURBIÈRES C., Femme en contextes : la conception stéréotypée du féminin au travers du langage documentaire (1958-2008), Mémoire d'habilitation à diriger des recherches en sciences de l'information et de la communication, Université de Toulouse, 2010.

COUZINET V., Médiations hybrides : le documentaliste et le chercheur en sciences de l'information, ADBS, Paris, 2000.

COUZINET V., «Vers une «société du savoir»: approche ethno-informationnelle de la «culture de l'information », Analele stiintifice ale universitatii Alexandru Ioan Cuza din Iasi [Annales scientifiques de l'Université de Iasi, Roumanie], vol. 1, n 1, p. 83-98, 2008.

COUZINET V., De la communication scientifique à la médiation spécialisée : communication des savoirs et formes d'hybridations, dans F. PAPY (dir.), Problématiques émergentes dans les sciences de l'information, Hermès, Lavoisier Paris, 2008a.

COUZINET V., «Représenter, répertorier, transmettre : formes d'institutionnalisation d'une discipline », dans R. M. MARTELETO et I. THIESEN (dir.), Mediações e Usos de Saberes e da Informação : um diàlogo França-Brasil, Actes du $1^{\text {er }}$ colloque du réseau MUSSI, Rio de Janeiro, 2008b.

COUZINET V., «Transmitir, difundir : formas de institucionalizaçao de uma disciplina », Perspectivas em ciencia da informaçao, vol. 14, nº spécial, p. 5-18, 2009. 
COUZINET V., «Janus ou le langage documentaire: union des deux faces d'un métier», dans I. FABRE (coor.), Professeur-documentaliste : un tiers métier, Educagri éditions, Dijon, 2011.

COUZINET V., «Objet de recherche et matériaux : les langages documentaires comme source et méthode pour les sciences de l'information et de la communication », Recherches qualitatives, h.s., nº 18, p. 27-38, 2016.

Delacheaume S., Guillaume J-C., «Espace forum », Journal de la psychanalyse de l'enfant, vol. 6, n 1, p. 217 226, 2016.

ECZET J-B., COMETTI G., «Est-il possible de faire le portrait d'un migrant ? Dilemmes anthropologiques », Terrain, 2019. doi : 10.4000/terrain.16129

Estivals R., «Classification de la bibliologie », dans R. Estivals (dir.), J. MEYRIAT (collab.), F. RichaUdEAU (collab.) Les sciences de l'écrit : encyclopédie internationale de bibliologie, Retz, Paris, 1993.

FABRE I., L'espace documentaire comme espace de savoir : itinéraires singuliers et imaginaires collectifs. Thèse de doctorat en Sciences de l'information et de la communication, Université de Toulouse 2, 2006.

FABRE I., VEYRAC H., «Des représentations croisées pour l'émergence d'une médiation de l'espace documentaire », Communication \& Langages, $\mathrm{n}^{\circ}$ 156, p. 103-116, juin 2008.

FABRE I., COUZINET, V., « Désir, curiosité, culture informationnelle : l'organisation des savoirs au cœur de l'histoire des idées », Revue Canadienne des sciences de l'information et de la bibliothéconomie, vol. 32, n 3-4, p. 85-105, septembre-décembre 2008.

FABRE, I., Médiation du contemporain : expérience esthétique de dispositifs documentaires. Retour réflexif sur les pratiques professionnelles. Habilitation à Diriger des recherches en Sciences de l'information et de la communication, décembre 2017.

FABRE I., GARDIES, C., «Questionner les représentations des usages d'un Learning Centre », dans Y. MAURY, S. Kovacs, S. CONDETTE (Dir.), Bibliothèques en mouvement : innover, fonder, pratiquer de nouveaux espaces de savoir, Editions du Septentrion, Lille, 2018.

FABRE I., GARDIES C., FAURE, L. « Appropriation d'un espace learning lab, étape d'une professionnalisation ? », dans Professionnalisation des acteurs des systèmes éducatifs. Cepadues éditions, juin 2019. (A paraître).

GARNIER F., Thésaurus iconographique : système descriptif des représentations, Le Léopard d'or, Paris, 1984.

GDOURA W., «La conception de la bibliologie chez Al-Kalkashandi (VXe siècle)», dans Actes du $9^{e}$ colloque de bibliologie (Tunis, 20-24 mars 1990), Tunis, IPSI, 1993.

Guibet LafaYe C., «Portraits de terroristes », Communication, vol. 34, $\mathrm{n}^{\circ}$ 2, 2017, doi : 10.4000/communication. 7354

Knowles, J. G., Cole, A. L., Handbook of the arts in qualitative research, Sage Publications, Los Angeles, London, New Dehli, 2008.

Lahire B., Debroux J., Denave S., Faure S., Glas A., Millet M., Renard F., Tralongo S., Portraits sociologiques. Dispositions et variations individuelles, Nathan, Paris, 2002.

LAVAUD M., «Envisager l'histoire littéraire », COnTEXTES, vol. 14, 2014, doi : 10.4000/contextes.5925

LAVOIE C., JONCAS J. A., «Le dessin-entretien : un outil de collecte de données innovateur et approprié auprès des communautés culturelles et linguistiques minoritaires », Recherches qualitatives, vol. 34, n 1, p. 97-121, 2015.

LEBAHAR J-C., Le dessin d'architecte : simulation graphique et réduction de l'incertitude, Editions Parenthèses, Roquevaire, 1983.

MAGUet F., «Le portrait de Che Guevara. Comment la parole vient à l'icône », Gradhiva, n 11, p. 140-161, 2010.

MAUDUIT C., « Portraits de paysans », Cahiers des études anciennes, LII, p. 47-69, 2015.

MKADA E-K., «L'organisation des connaissances dans les thésaurus : une approche pragmatique cognitive », Études de communication, $\mathrm{n}^{\circ} 39$, p. 139-154, 2012.

MEYRIAT J., «Pour une classification des sciences de l'information et de la communication», Schéma et schématisation, $\mathrm{n}^{\circ} 19$, p. 61-64, 1983. 
MEYRIAT J., «La bibliologie parmi les sciences de l'information et de la communication », dans R. ESTIVALS (dir.), J. MEYRIAT (collab.), F. RichaUdEAU (collab.), Les sciences de l'écrit : encyclopédie internationale de bibliologie, Retz, Paris, 1993.

NANCY J-L., Le regard du portrait, Galilée, Paris, 2000.

NGUYEN K-C., «Le dessin de la Personne », dans La personnalité et l'épreuve de dessins multiples. Maison, arbre, deux personnes, Presses Universitaires de France, Paris, 1989.

OCHANINE D., Kozlov V., «L'image opérative effectrice », Questions de psychologie, $\mathrm{n}^{\circ}$ 3, traduit dans L'image opérative, Actes d'un séminaire et recueil d'articles de OcHANINE D., p. 225-251, 1971.

PINCEMIN B., «Interprétation, contextes, codage », Corpus, n 6, p. 5-15, 2007.

REBHi M., Odoura W., « Deux précurseurs de la bibliologie en terre d'Islam », Communication et langages, $\mathrm{n}^{\circ}$ 86, $\mathrm{p}$. 5-21, 1990.

RÉGIMBEAU G., "Clés d'accès aux images fixes : indexation et perspectives pédagogiques à partir des ressources d'Internet », Spirale, n 28, p. 233-251, 2001.

RUEDA A., «Du portrait cinématographique documentaire au portrait en sciences de l'information et de la communication », Sciences de la société, nº 92, p. 177-191, 2014.

SALDANHA G., «Un portrait de Robert Estivals au miroir de la Revue de Bibliologie : des chemins théoriques pour une réflexion historique », Les Cahiers du numérique, (en cours de parution).

Schmitт F., SCHмiтt P., « Portrait de l'ethnographe en artiste », Revue des Sciences Sociales, n 56, p. 82-95, 2016.

SÉNÉQUIER A., FALEH S., JouSSELME C., «Atelier portrait : médiation artistique pour une reconstruction narcissique dans un contexte de blessé de guerre », Annales Médico-Psychologiques, n 173, p. 25-30, 2015.

SERVICE JURIDIQUE ET TECHNIQUE DE L'INFORMATION ET DE LA COMMUNICATION, COMMISSION DE LA CARTE D'IDENTITÉ DES JOURNALISTES PROFESSIONNELS, MINISTÈRE DE LA CULTURE ET DE LA COMMUNICATION, INSTITUT FRANÇAIS DE PRESSE, Les Journalistes français en 1990: radiographie d'une profession, La Documentation française, Paris, 1991.

SOUMAGNAC-COLIN K., « Construire l'interopérabilité culturelle autour de la documentation et de l'information dans une communauté de pratique », Communication, vol. 34, $\mathrm{n}^{\circ}$ 1, 2016, doi : 10.4000/communication.6670

StOCK C., MARES S., Robinson G., «Telling and re-telling stories : the use of narrative and drawing in a group intervention with parents and children in a remote aboriginal community », The Australian and New Zealand Journal of Family Therapy, vol. 33, n² 2, p. 157-170, 2012.

VÉRON E., Espaces du livre : perception et usages de la classification et du classement en bibliothèque, Bibliothèque publique d'information, Paris, 1989.

Veron E., LeVASSEuR M., Ethnographie de l'exposition. L'espace, le corps et le sens, Bibliothèque publique d'information, Paris, 1990.

Wrona A., «Des Panthéons à vendre : le portrait d'homme de lettres, entre réclame et biographie », Romantisme, vol. $1, \mathrm{n}^{\circ} 155$, p. 37-50, 2012.

Zakhartchouk J-M., «Consignes : aider les élèves à décoder », Pratiques : linguistique, littérature, didactique, ${ }^{\circ}$ 90, p. 9-25, 1996. 